\title{
An improved Whale Optimization Approach for Effective Data Transmission for IoT Communication
}

\author{
Abidullha Adel, Md Sohel Rana, Md Nuruzzaman Rana, Md Alamin Hossan, \\ And Mohammad Akbar Shahpoor
}

\begin{abstract}
Internet of Things (IoT) offers interconnection among several wireless communication devices for the provision of device accessibility and in-built capacity. IoT provides device interaction and provision of advantages capability for networking and socialization with consideration of intermediate devices. Through innovation in technology IoT devices convert cyber environments with hyper-connectivity. IoT communication contains several smart devices such as body sensors, smartphones, tags, electronic gadgets, and so on. IoT communication is involved in the provision of heterogeneous connectivity among devices for the provision of interface and connectivity for enhancing service quality. The data sending among IoT devices is affected by several threats that have an impact on the network's performance. To overcome the limitation related to IoT communication, it is necessary to develop an appropriate technique for enhancing IoT network communication performance. In this research developed a multi-channel routing approach is adopted in IoT communication. The developed approach utilizes a metaheuristics approach with probability-based characteristics. For the meta-heuristics approach this research utilizes whale optimization technique combined with probability characteristics for improving the IoT communication performance of the network. The proposed approach utilizes initially constructs the IoT communication path for information sharing and gathering. This path information is identified through the objective function of a meta-heuristic approach. Based on the objective function hoping between the devices is minimized through which data are transmitted in the network. Simulation is performed as a unique proposed approach with a coverage area of $\mathbf{1 0 0}$ meters. For identification of the optimal path in the network, WOA identifies the path of communication through probability function. Comparative analysis of research exhibited that WOA provides significant performance with the identification of optimal value at the range of 1.0746e-78. Further, the proposed probability-based WOA approach significantly improves the performance of the IoT network.
\end{abstract}

Keywords - The Internet of Things (IoT), Meta-heuristics, Whale Optimization, Multi-path, Optimal path.

\section{INTRODUCTION}

The Internet of Things (IoT) provides interconnection of variant wireless communication devices which offers ubiquitous accessibility of devices and in-built intelligence capacity. IoT offers interaction with devices and provides sufficient capability advantages of networking and socialization with consideration of intermediate devices. Emerging innovation in IoT devices transforms the lives in the cyber-physical-social environment through hyperconnectivity [1]. Devices such as smart tags, body sensors, embedded objects, smartphones, bio-Nano-things, and conventional electronic gadgets. IoT devices provide heterogeneous connectivity to withstand interface and connectivity demand to improve the quality of service [2]. IoT communication is categorized as the Social Internet of Things (SIoT), this belongs to the specialized IoT subset. Gene rally, IoT provides heterogeneous communication among devices to perform a specific task through collaboration and socialize approach. Due to an emerging interest in the IoT research domain mobility management protocols are arisen to withstand the present environment. The nodes of IoT devices are a growing area of interest domain and it leads to the development of routing management protocol has attracted the attention of researchers. The characteristics static of nodes is not dominant. The provision of access to information requires the routing management protocol for the identification of user location. The devices in IoT are moving within the infrastructure or outside the infrastructure [3].

\section{LITERATURE REVIEW}

Multipath routing involved in the demonstration of the efficiency of routing through improving the performance of the IoT network in which alternate paths are identified between source and sink. This technique needs to cope with the existing solution for consideration of different limitations [4].

Several factors are involved in IoT design protocol for routing those protocols are presented and explained in the following section [5].

In IoT, devices data are generated expired within a specified time since it is required for data transmission from source to a destination within the stipulated period. It is necessary to handle the latency of protocol routing for the appropriate maintenance of service quality [6].

\footnotetext{
Nuruzzam Rana, Daffodil International University, Bangladesh. (e-mail: Nuruzzamanrana1 ${ }^{\circledR}$ gmail.com) Md Alamin Hosan, Daffodil International University, Bangladesh. (e-mail: alamin311293 @ gmail.com) Mohammad Akbar Shapoor, Kunduz University, Afghanistan. (e-mail: akbarshshpoor@gmail.com)
}

\footnotetext{
Submitted on November 15, 2021

Published on December 16, 2021.

Abidullha Adel, Nanjing University of Information Science and

Technology, China, Afghanistan.

(e-mail: abidullhahadel111@gmail.com)

Md Sohel Rana, Nanjing University of Information Science and

Technology, China, Bangladesh.

(e-mail: sohelrana117373@gmail.com)
} 
The IoT device protocol utilizes a single path while other paths are kept as backup else those paths are used as multipath for round-robin fashion which involves the establishment of the path in a single time [7].

The distribution of traffic in the IoT devices is not equal for all established links in the network, for multiple routes traffic are spreading in all routes which alleviate the device congestion in the links and bottlenecks [8].

\section{RELATED WORKS}

Mauro A. A. da Cruz [9] presented research for opensource middleware for evaluation of proprietary solutions such as Intel for the Intel Smart Campus with consideration of different performance metrics. The evaluation is performed based on the middleware. Results stated that developed metrics perform effectively and considered as middleware for IoT solutions. The middleware offers significant performance through the proposed approach.

Jilong $\mathrm{Li}$ [10] proposed a scheme for minimizing end-toend delay in the IoT network. This study focused on the quality of network from source to destination node at expected IoT communication lifetime through consideration of IoT heap traffic in the network. The proposed approach effectively broadcasts RREQ between clusters of flooding. Through the proposed scheme RREQ overhead and search time for a path are minimized and improves the efficiency of the network transmission rate. The simulation results demonstrated Since the suggested method decreases channel congestion interference and information queue. Further the simulation results demonstrated the method scheme performs effectively rather than existing approaches. But this research fails to provide the amount of performance enhancement through the proposed scheme.

Dmitrii Chemodanov [11] presented an Artificial Intelligent (AI) for consideration of different geographic routing approach. The proposed scheme relies on the imagery of satellite through the adoption of deep learning techniques. The proposed scheme utilizes a greedy forwarding mechanism which minimizes local minimum problem with diversified traffic in the network which emulates electrostatic repulsive forces. The theoretical analysis of results demonstrated that Greedy forwarding provides an approximation bound of 3.291 as the shortest path value duct consideration of unit disk graphs or symmetrical links the network. The simulation results of both event-driven approach and numerical values involve incident-supporting hierarchical approach for improving the throughput of the network to reduce failure of devices and challenges related to mobility management. However, this research fails to provide a mathematical explanation of the IoT devices.

Zhijie Han [12] proposed an approach for application of IoT cloud specifically for Healthcare applications. This research proposed approach combines the concept of Trie and hash methods. The proposed algorithm is named as hash offset Trie match on the longest prefix (HOTMLP) algorithm. The proposed HOTMLP divides the longest prefix in the routing table where common prefix is identified in the routing table. Route prefixes are placed in the hash table to reduce step size and storage space.. Through the application of the Trie method prefix matching entry will be identified.
Analysis of the proposed approach demonstrated that it exhibits several advantages such as faster, ease of update and minimal storage space. The proposed prefix involves longer prefix matching complexity with the order of $\mathrm{O}(2+n / k)$. In other cases, it uses " $n$ " is 8 for IPv4 address is 16 and IPv6 address is 32. Experimental results analysis illustrated that the proposed approach performs effectively based on the core routing approach with improved lookup efficiency and forward tens and millions of packets per second [7].

Luca Calderoni [13] discussed several IoT platforms specifically for the management of the network. The proposed system involves the scientific community which concerns IoT solutions. Results demonstrated that LGPL offers significant testbed for IoT manager. This research fails to provide a detailed mathematical explanation about the proposed concept.

Soobin Jeona Inbum Jung [14] proposed an approach named as MinT Improved (MinT-I) for improving the performance of middleware for thread adjustment. The performance is aimed to enhance the performance through different connection parts concerning retransmitting, packet reception, analyze and processing. Experimental results illustrated that the proposed MinT-I approach improves the throughput effectively at the rate of $25 \%$ to $35 \%$ for middleware. But the proposed MinT-I approach focused on resource usage and memory optimization. Further the proposed approach reduces power consumption level and latency of IoT devices.

Sankar Mukherjee \& G.P. Biswas [15] offered a whole solution that covers network protocols, spectrum distribution, node deployment, MANET routing, and mobility pattern, as well as the implementation of IoT applications that are simulated using the Omnet++ simulator and demonstrated to be feasible and perform well.

ValentinaCasola et al. [16] proposed an approach for supporting the IoT system with an appropriate security analysis scheme. The proposed model composes of an automated process for modeling threats and assessment of risk through the identification of a control scheme for implementing prior security factors. The effectiveness and application are adopted for an automated system for commercial products of IoT applications.

Morteza Safaei Pour [17] determined the magnitude involved in the exploitation of IoT applications. Also, this research examined the backbone of operator network and Internet Service Providers (ISPs) based on the geolocation database with consideration of the environment in hosting with the classification of an inferred device of IoT based on the consideration of hosting sector especially manufacturing sector. The results demonstrated that the IoT system is subjected to an insecurity paradigm and it is a landscape for IoT applications.

Mahmudul Hasan [18] evaluated different machine learning models involved in anomaly and attack prediction of the IoT system. The machine learning Logistic Regression (LR), Support Vector Machine (SVM), Decision Tree (DT), Random Forest (RF), and Artificial Neural Network (ANN) are the algorithms studied in this study (ANN). Recall, precision, accuracy, F1 score, and area under the Receiver Operating Characteristic Curve are among the measurements assessed. For classifiers like random forest, decision tree, and 
knapsack, the accuracy value is $99.4 \%$ and ANN. This technique has similar accuracy while comparative analysis stated that Random Forest exhibits significant performance.

\section{Proposed Methodology}

This research aimed to improve the performance of IoT network communication hence the adopted meta-heuristics approach for performance enhancement. this section presented the proposed multi-channel approach for performance enhancement of IoT communication. This section also presented the proposed methodology for performance enhancement of the IoT communication along with the software used, phases of research and expected outcome. This chapter presented the basic concept related to the routing strategy and proposed meta-heuristics approach.

\section{A. Routing Performance Measures in IoT Communication}

As stated by the name Internet of Things composes of two things such as Internet" and Things". Here the first terms denote the aspect of the network -orientation where servers of the Internet are interconnected through the central building for computing device communication. This requires an effective for contexts such as Urban-Low Power, Lossy Networks (LLNs), Building Automation, and Home Automation, a routing protocol is used. Despite the fact that these domains are divided into categories due to differences in pattern, network size, and mobility. The following are the primary limits of IoT communication that are stated and classified as a result of this:

\section{B. Traffic Patterns IoT}

$\mathrm{T}$ routing pattern need to match with the traffic and deployment area of the network. No need every protocol needs to be performed efficiently but it requires a single appropriate protocol for deployed IoT communication network.

\section{Energy Efficiency}

IoT devices are deployed based on the battery-driven for an extended period with consideration of IoT communication. Routing protocol with efficient energy consumption functionality is considered as a major function of IoT communication. Based on the node of constraint communication higher rate of information can be collected in the network.

\section{Scalability}

This IoT protocol stack ranges from 100 to $1,000,000$ nodes, which requires significant performance and memory usage. IoT network increase in size subjected to increases in table size for routing.

\section{E. Mobility}

IoT communication does not subject to mobility often even it requires an appropriate routing scheme for changes in node location. Additionally, IoT subjected to unique challenges for offering significant performance.

\section{F. Directionality}

IoT communication not able to cope with the wireless network and bi-directional connectivity among nodes.
Routing protocol offers identification of IoT links and identifies the best path for transmission.

\section{G. Transmitter Usage}

With the consideration of energy consumption rate is higher for constrained devices. Hence, the IoT communication mainly relies on a sparse network.

Based on the consideration of the above parameters into consideration this research proposed an effective routing approach.

\section{H. Existing Method}

The Internet of Things is a large network that network devices allow information to be transformed without the need for human contact. This allows for remote object management by allowing interaction with the physical environment. The Internet of Things can be used in a vast number of applications. such as semantic, wireless sensor networks, data modeling, cloud computing, processing, and so on. Generally, IoT devices are heterogeneous in nature since it includes various functionalist, resolution, and operating condition, and so on. Existing routing techniques include a decentralized approach for controlling energy which involved the transmission of energy. This utilizes cluster tree topology for the construction of the IoT network through location and data sensing data can be transmitted through the network. The constructed tree collect data from different sources and delivers data. Even this approach includes the construction of spanning-tree this forwards data to the destination node but data transmission over the network using constructed tree vein the energy level of the IoT network. The other techniques utilized in IoT for data transmission are:

\section{Cluster-based Routing}

This is predicated on the use of a spanning tree to transmit data from source to destination. Frequent communication among devices, on the other hand, causes energy loss in the network.

\section{J. Context-primitive Methods}

This approach involved in the transmission of data through the ranking process among nodes. Also, it classifies the entire region categories, but this approach is not appropriate for a large area.

\section{K. Centralized Routing}

Based on the formulation of routing table data are transmitted between senders to the receiver. Node shortest paths are estimated using a sink node also sink node capable for maintaining network topology. This technique is not effective due to increased routing overhead.

Hence, there is a lot of demand for improving the performance of IoT communication since existing techniques are limited to certain constraints. The developed approach uses a multi-channel routing technique for improving the performance of IoT communication. The developed approach includes the construction of IoT network with an estimation of node information, once information about IoT nodes is estimated multi-path-based optimization is employed for estimation of an effective path for data transmission in the 
network. Some other existing techniques included for routing in IoT communication are shown in below table:

TABLE I: EXISTING TECHNIQUES

\begin{tabular}{|c|c|}
\hline Method & Steps \\
\hline $\begin{array}{lr}\text { Hierarchical cluster- } \\
\text { based routing } \begin{array}{r}\text { with } \\
\text { decentralized }\end{array} \\
\text { efficiency }\end{array}$ & $\begin{array}{l}\text { Based on the residual energy of the } \\
\text { device's data are transmitted in the } \\
\text { network for transmission of data }\end{array}$ \\
\hline $\begin{array}{l}\text { Velocity } \quad \text { Cluster } \\
\text { (VLECET) }\end{array}$ & $\begin{array}{l}\text { Tree topology is adopted for data } \\
\text { transmission among the cluster network. }\end{array}$ \\
\hline $\begin{array}{l}\text { Relay chain routing that } \\
\text { adapts }\end{array}$ & $\begin{array}{l}\text { Load balancing is applied for the } \\
\text { estimation of relay nodes in the network }\end{array}$ \\
\hline $\begin{array}{l}\text { Mobile Data Gathering } \\
\text { using Bounded Relay } \\
\text { Hops (BRH-MDG) }\end{array}$ & $\begin{array}{l}\text { Data are gathered and transmitted to the } \\
\text { destination node using a polling } \\
\text { approach. }\end{array}$ \\
\hline $\begin{array}{lr}\text { Cluster-Based } & \text { Routing } \\
\text { with Low Energy } \\
\text { Consumption }\end{array}$ & $\begin{array}{l}\text { This transmits data in a cluster network, } \\
\text { where devices are grouped as different } \\
\text { clusters and data can be transmitted } \\
\text { between those clusters. }\end{array}$ \\
\hline Primitive method & $\begin{array}{l}\text { Through ranking nodes in IoT, network } \\
\text { data are transmitted }\end{array}$ \\
\hline
\end{tabular}

\section{Flow Chart of Proposed}
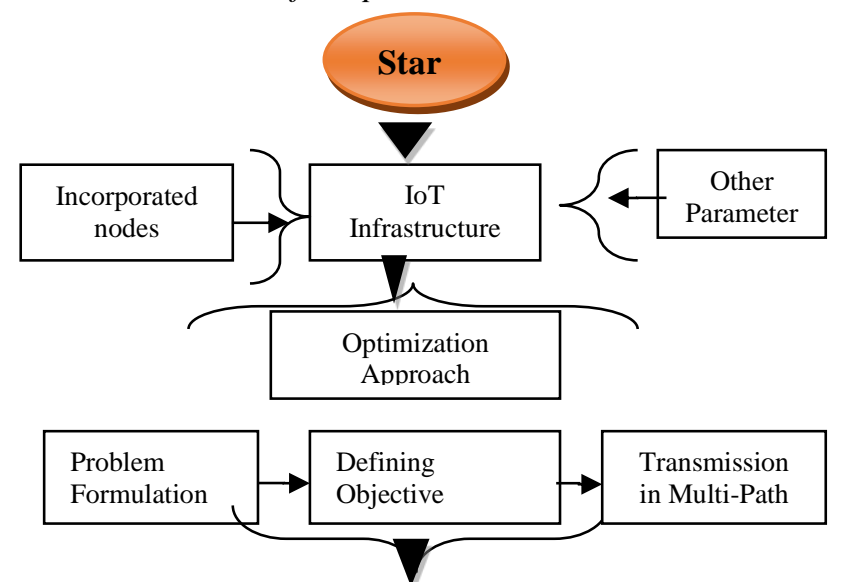

Ontimal Path Identification I Jsino Multi-Channel Routino

Identification of Optimal/Effective path for IoT communication

Evaluation of Parameters Using Game Theory Approach
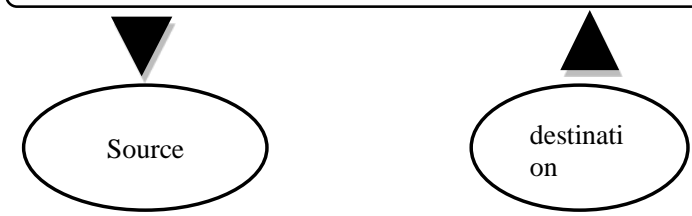

\section{Proposed Methodology}

To improve the performance and adopt significant routing in IoT communication this research uses meta-heuristicsbased optimization known as Whale optimization through a multi-channel routing approach. In the proposed technique IoT devices are arranged in mesh form and transmission is performed between IoT devices. In the framed IoT devices spectrum optimization approach through a heuristic algorithm is adopted for transmission of data from the devices to devices in the IoT system. For analyzing every device detail involved in IoT connectivity, the proposed framework employs an optimization technique. The optimization method considers the distance between IoT devices, energy consumption, device routing, and other factors. After the evaluation of all network parameters of IoT devices efficient routing path is evaluated through the consideration of energy level, multiple routing paths are identified through an optimization algorithm that offers a significant path. Through the identified path data are transmitted between source devices to destination devices for effective transmission. This proposed multi-channel routing with optimization approach expected to improve the performance of the system.

\section{N. Whale Optimization Approach}

Whales are the world's largest mammals, measuring 30meters in length and weighing 180 tons, as we all know. Killer whales, Minke whales, Sei whales, humpback whales, right whales, finback whales, and blue whales are among the seven kinds of whales. Whales are commonly seen as predators.A mathematical model for the meta-heuristics approach is constructed and its performance is presented based on this presented:

\section{O. Encircling Prey}

Humpback whales are capable of locating their prey and encircling them. Because the search space's character is uncertain. The agent's stance is given as follows, based on the evaluation of the intended prey:

$$
\begin{aligned}
& \tilde{D}=|C \vec{X}(t)-X(t)| \\
& \vec{X}(t+1)=\vec{X}(t)-\vec{A} \cdot \vec{D}
\end{aligned}
$$

where tshowthe current iteration, A, C show coefficient vectors, and $\mathrm{X}$ show the significant solution for vector position identification.

This vector can be calculated using the following formula:

$$
\begin{aligned}
& \vec{A}=2 \vec{a} \cdot \vec{r}-\vec{a} \\
& \vec{C}=2 \cdot \vec{r}
\end{aligned}
$$

In the preceding equation, the number of iterations decreases linearly. This is taken into account while evaluating the exploratory phase.

\section{P. Proposed Multi-Path Optimization (Probability Whale Optimization Approach)}

The multipath routing strategy entailed identifying alternate paths for each network node's destination node. With a higher frequency of nodes in the network, the cost of data transmission must be higher. Routing decisions are monitored via the determination of the network's probabilistic value by including a probability model.

$$
G(P)=\left\{\begin{array}{l}
1 p \leq p_{0} \\
0 p>p_{0}
\end{array}\right.
$$

The value 1 denotes the connection is successful and the average path of the IoT communication is represented as,

$$
f\left(v_{i}\right)=\frac{1}{\sigma \sqrt{2 \Pi}} e^{-\left(p_{i}-p_{\text {avg }}\right)^{2} / 2 \sigma^{2}}
$$


$2 \sigma^{2}$ reflects the IoT communication path's variance and

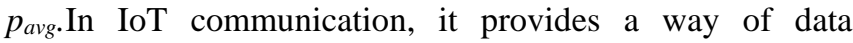
transmission.

The most efficient path for information communication in the IoT network is calculated using,

$$
E(N)=\rho d \lambda
$$

The packet transferred and other parameters are displayed in this number of nodes.

\section{EXPERIMENTAL RESULTS}

Influenced by a variety of elements, one of which is the network The purpose of this research was to use an effective routing approach to improve the communication performance of IoT devices. The challenges associated with IoT communication and threats are presented in the existing chapter. This research offered an effective multi-channel routing technique for increasing the performance of the IoT communication system utilizing a heuristic approach to tackle the obstacles associated with IoT communication. The heuristic approach is employed for the optimization of IoT network performance. The optimization algorithm implemented in this research is whale optimization which involved in improving the performance of IoT communication.

The results acquired using the proposed optimization approach were provided in this chapter. The following is how the current chapter is structured: The first segment discussed the development of the IoT network and followed by communication established in IoT communication. In next section presented about multi-channel communication performed in IoT communication. Finally, this section offers the overall performance of the proposed approach with other existing approaches.

\section{A. Proposed Optimization Algorithm}

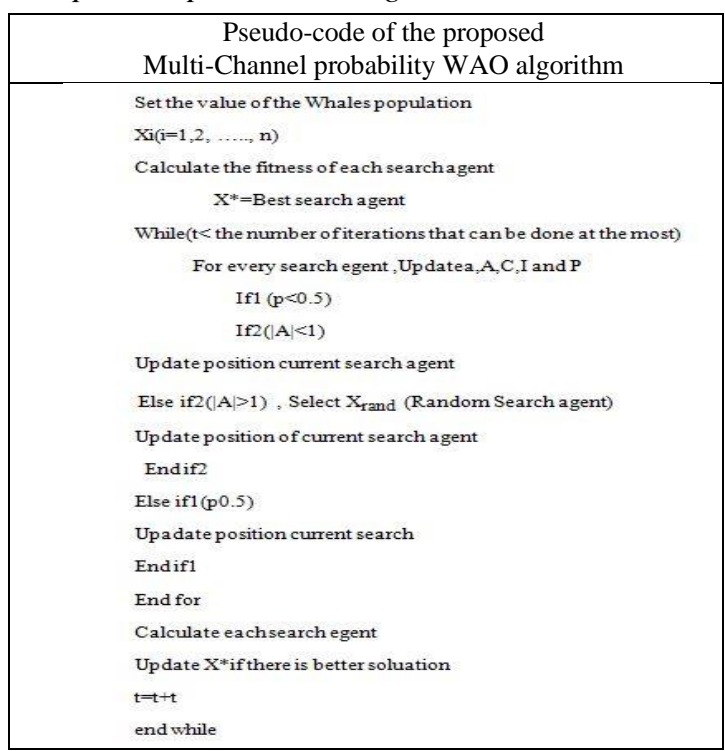

\section{B. Construction of IoT Network}

The data has been transmitted in the form of wireless communication in which data has been transmitted among nodes or IoT devices. The proposed heuristics approach objective function is to enhance the performance of IoT communication. The below Fig. 1 illustrates the construction of IoT communication in which 50 devices are constructed. The constructed IoT devices are deployed in the coverage area of 100meters.

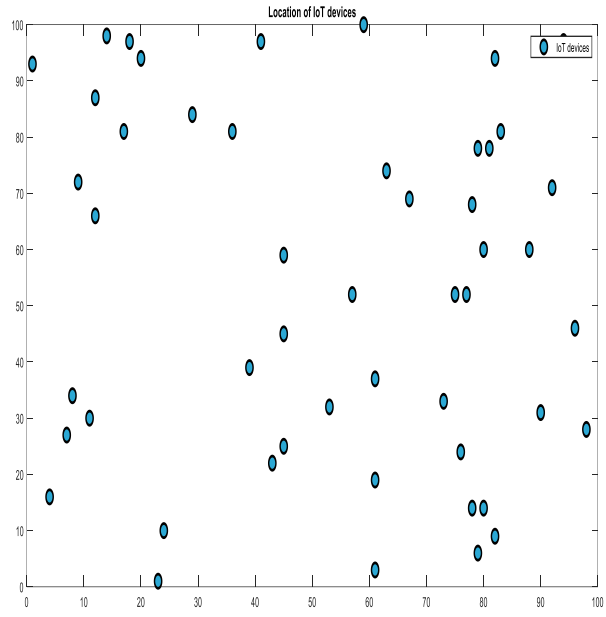

Fig. 1. construction of IoT communication.

The below Fig. 2 provides data communication performed among IoT communication. The established IoT infrastructure consists of 50 nodes and data were transmitted through other nodes of IoT devices in circular form. Data collected through IoT communication improves the communication range of IoT devices with improved communication range. Constructed IoT network involved in improved communication range of 145 meters of communication range.

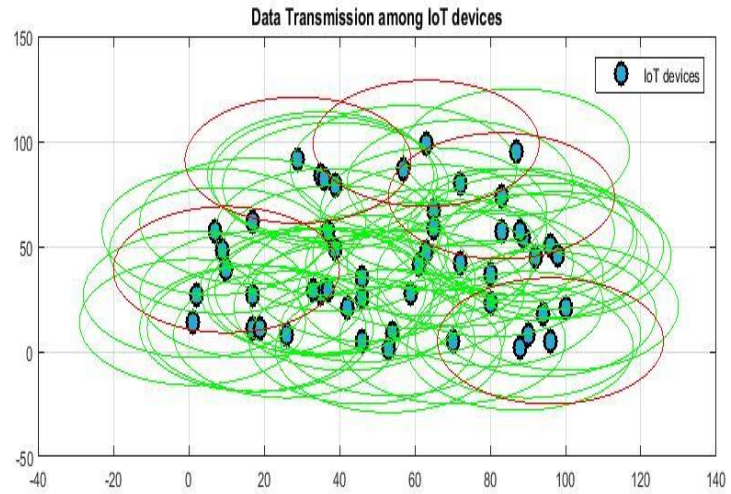

Fig. 2. Data communication performed among IoT

In the above figure the red circle indicates a higher interference communication channel for data transmission using IoT communication.

\begin{tabular}{cc}
\multicolumn{2}{c}{ TABLE II: CONSTRUCTION PARAMETERS } \\
\hline Parameters & Values \\
\hline IoT devices & 50 \\
Communication Range & 100 meters \\
Data communication range & 145 meters \\
\hline
\end{tabular}

The above table also illustrates the constructed communication network among IoT devices data in tabular form. 


\section{Probability Whale Optimization Approach}

Meta-heuristics algorithm involved mimicking the behavior of the whale's humpback which involved in optimization of the device location. WOA algorithm is based on the hunting behavior of the optimization of IoT devices which exhibits the hunting behavior of the whale with a bubble net attacking mechanism for identification of optimization value of the proposed approach. The multichannel WOA provides a comparative advantage over other optimization techniques with improved network performance of the network. The below Fig. 3 provides the coverage range of the proposed multi-channel routing through the proposed WOA approach in the 3-D plot. The 3-D graphical representation of WOA offers an IoT communication scenario.

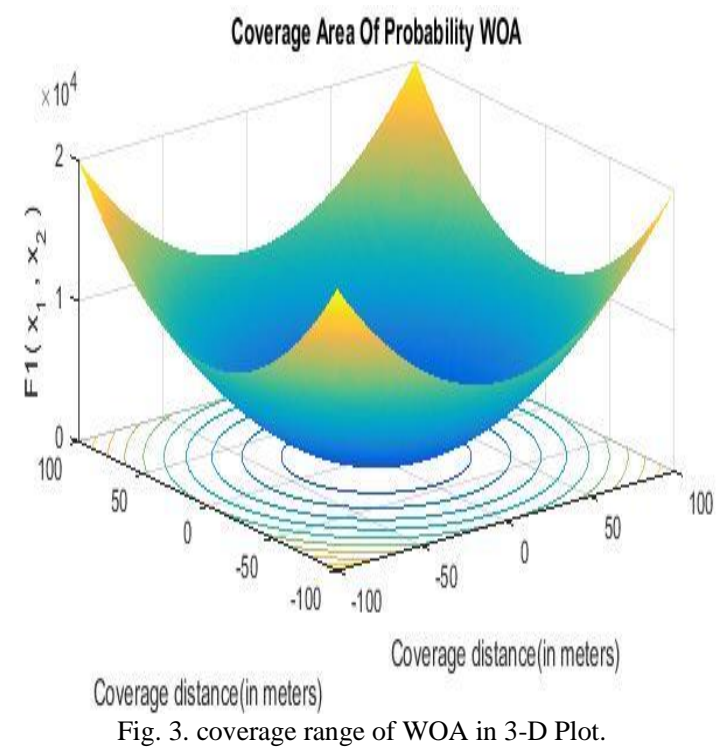

The above Fig. 3 3-D plot demonstrates the coverage range obtained by the proposed multi-channel WOA approach. The routing of network the IoT devices is enhanced by the developed WOA approach since the designed IoT infrastructure involved in the coverage range of $100 \mathrm{~m}$. The proposed multi-channel WOA provides coverage range of $100 \mathrm{~m}$ in both $\mathrm{x}$ and $\mathrm{y}$-direction which means it significantly perform with IoT infrastructure. Even in z-direction also the coverage is obtained as $200 \mathrm{~m}$ hence the WOA improves the coverage efficiently. Further at the edges also the performance of IoT devices is higher for which means it has minimal interference rate and it will effectively enhance the data transmission scenario of IoT device communication.

In the meta-heuristics approach optimization algorithm, the main functionality is the evaluation of effective objective function. To improve the performance of multi-path communication among IoT devices WOA involved in the identification of optimal solution through which the data in the IoT network infrastructure that has been built is performing well. Value optimization in the development of an effective optimal path for data transfer among IoT network architecture. Using the identification of optimal values data in the network improves the performance of the constructed network.

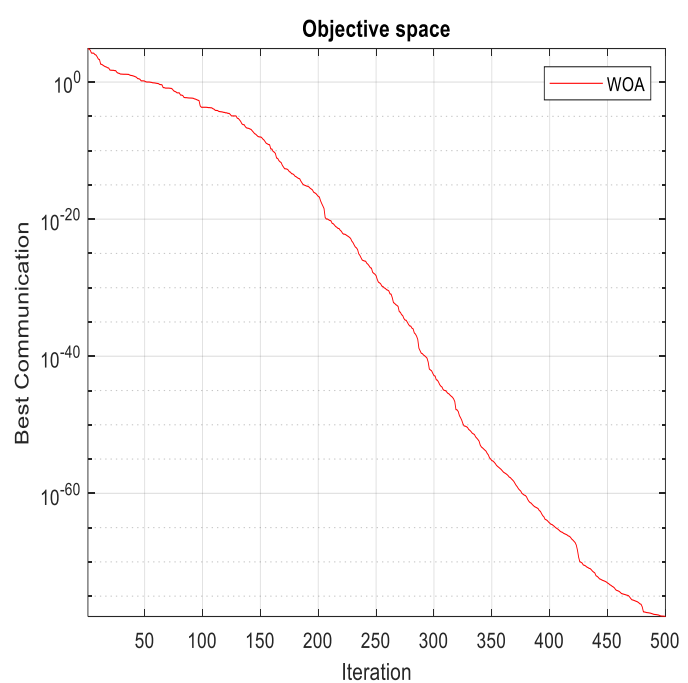

Fig. 4.Optimal value for data transmission among IoT devices.

Fig. 4 provides the optimal value for data transmission among IoT devices. The designed multi-channel WOA algorithm is evaluated for 500 iterations where efficient communication among devices is observed at the transmission range of 10 to $10 \mathrm{e}-60$. In Fig. 4 even the communication is obtained as $200 \mathrm{~m}$ in z-direction similar manner 7 also illustrates the efficient path for the establishment of communication among IoT devices for improved communication performance among IoT devices. The optimal solution obtained by the multi-channel WOA presented in the below table.

\begin{tabular}{cc} 
TABLE III: OPTIMAL SOLUTION \\
\hline Optimal Solution Obtained by WOA \\
\hline $2.2194 \mathrm{e}-40$ & $-7.2417 \mathrm{e}-41$ \\
$2.5912 \mathrm{e}-40$ & $6.4058 \mathrm{e}-42$ \\
$-8.3253 \mathrm{e}-41$ & $-3.1569 \mathrm{e}-41$ \\
$6.3461 \mathrm{e}-41$ & $-8.2749 \mathrm{e}-41$ \\
$8.8161 \mathrm{e}-41$ & $-6.2968 \mathrm{e}-40$ \\
$1.1528 \mathrm{e}-40$ & $2.0169 \mathrm{e}-40$ \\
$-7.3556 \mathrm{e}-41$ & $-1.1542 \mathrm{e}-40$ \\
$6.4877 \mathrm{e}-41$ & $-4.8099 \mathrm{e}-41$ \\
$-3.781 \mathrm{e}-40$ & $2.231 \mathrm{e}-40$ \\
$5.8582 \mathrm{e}-41$ & $5.9911 \mathrm{e}-41$ \\
$1.3771 \mathrm{e}-40$ & $-5.9152 \mathrm{e}-41$ \\
$-2.4907 \mathrm{e}-40$ & $2.0032 \mathrm{e}-40$ \\
$-6.4648 \mathrm{e}-41$ & $-1.1137 \mathrm{e}-40$ \\
$-1.5285 \mathrm{e}-40$ & $-2.7656 \mathrm{e}-40$ \\
$-9.834 \mathrm{e}-41$ & $1.133 \mathrm{e}-41$ \\
\hline
\end{tabular}

The above table provides an optimal value identified for the developed multi-channel WOA algorithm. Optimal value identified are 15 values where the data can be evaluated for identification of optimal value for the metaheuristics approach. For the identified values IoT network provides efficient network performance for improving IoT device communication. Through the proposed approach optimal value identified for the WOA approach is identified as $1.0746 \mathrm{e}-78$ which is considered as an optimal value for the developed IoT infrastructure. 


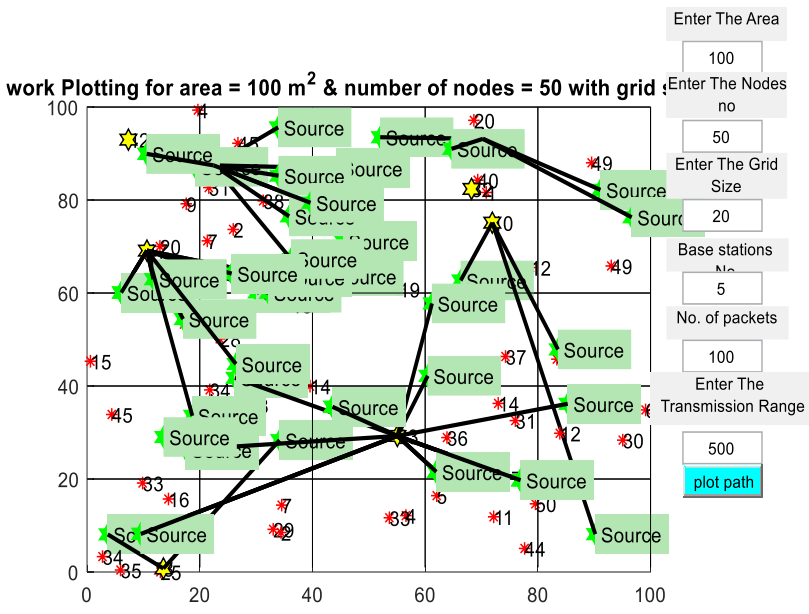

Fig. 5. IoT device communication.

Fig. 5 provides the IoT device communication for the constructed device infrastructure. The optimal location identified for device communication is evaluated using simulation software. The evaluation of communication is performed through the constructed network for the transmission range of $500 \mathrm{~m}$ which has a base station count of 5 for the area location 100. For evaluation grid size considered are 20 with a packet count of 100 which means it significantly transmit the data among IoT devices effectively? the transmission range considered for analysis is 500 meters with packet count 100 with the coverage area of 100 meters with consideration of 50 IoT devices.

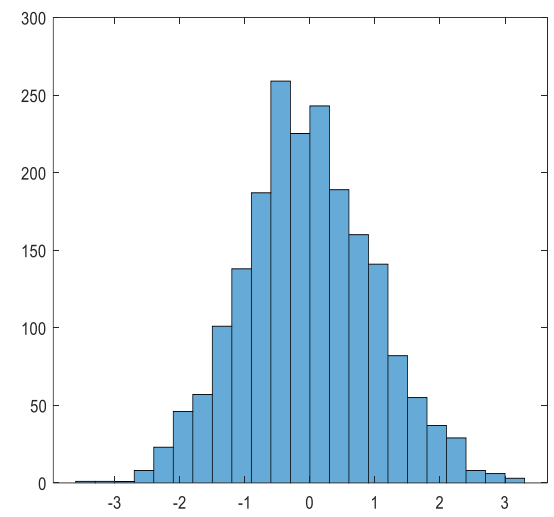

Fig. 6. IoT devices probability distribution.

The probability of IoT device used for the establishment of communication among IoT devices are demonstrated in Fig. 6. The IoT devices probability distribution is shown in the figure for the range of infinity which has the effective value range of 300 with significant performance. The analysis of device probability is effective rather than other approaches for the constructed IoT devices infrastructure.

\section{Comparison of Proposed Vs Existing}

The proposed multi-channel optimization algorithm has been examined effectively through comparison with another optimization algorithm.

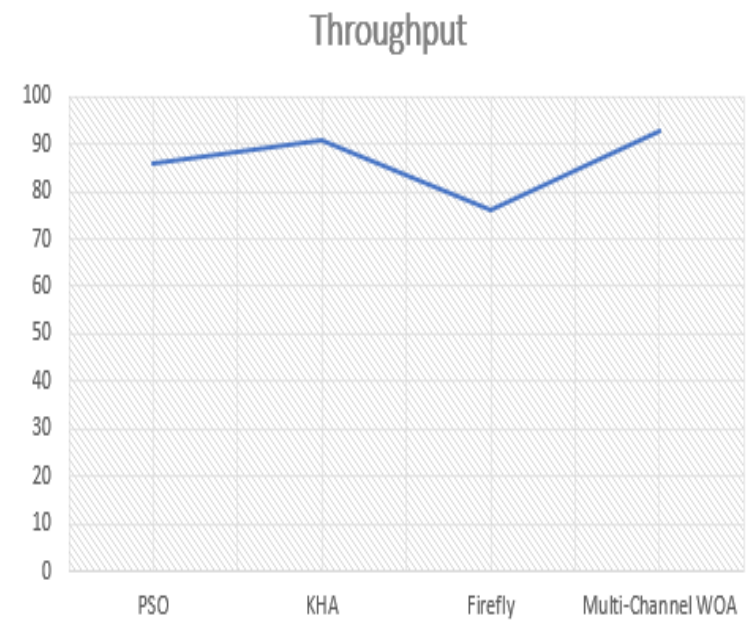

Fig. 7. Throughput calculation of proposed approach.

Fig. 7 shows a comparison of the suggested approach's throughput calculation with that of other known optimization approaches. Figure shows that firefly optimization, PSO, and KHA yield minimal throughput values of 76, 86, and 91, respectively, however the suggested multi-channel WOA delivers a throughput value of 93 , which is much greater than the existing methodologies.

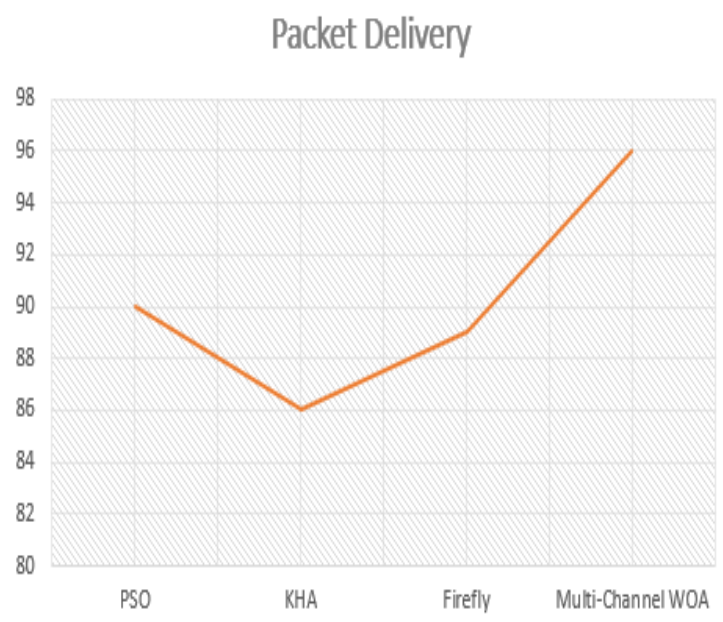

Fig. 8. The proposed approach's packet delivery rate.

The packet delivery rate analysis of the proposed approach with existing approach stated that proposed approach performs effectively rather than other approached since the firefly algorithm provides the optimal value of 89 , PSO value of 90 and firefly provides a delivery ratio of 89 in which proposed multi-channel WOA offers delivery ration of 96 . This is a substantial improvement over current method. The proposed approach's performance is compared to that of another optimization approach, which is shown in tabular form as follows:

\begin{tabular}{ccc}
\multicolumn{2}{c}{ TABLE IV: COMPARISONS OF PROPOSED VS EXITING } \\
\hline Optimization Approach & Throughput & $\begin{array}{c}\text { Packet Delivery } \\
\text { Ratio }\end{array}$ \\
\hline PSO & 86 & 90 \\
KHA & 91 & 86 \\
Firefly & 76 & 89 \\
Multi-Channel WOA & 93 & 96
\end{tabular}


The above table provides a comparative analysis of comparing the proposed optimization strategy to other optimization algorithms that are already in use The proposed optimization algorithm is compared to existing approaches in a comparative study. In comparison to other existing techniques, WOA provides significant throughput and packet delivery ration performance.

\section{E. Finding of Research}

For data transmission from source to destination, IoT connectivity necessitates multi-path communication. Higher energy levels are required for optimal data transfer between devices. As a result, this study employed whale optimization and uses a probabilistic routing strategy to convey data across IoT devices while reducing traffic overhead. For decisionmaking, a prediction of the target node was done. The proposed meta-heuristics-based optimization approach exhibits significant functionality for consideration of objective function. To improve the performance of multi-path communication among IoT devices WOA involved in the identification of optimal solution through which data in the constructed IoT network infrastructure perform effectively. The proposed multi-channel WOA provides coverage range of 100 meters in both $\mathrm{x}$ and y-direction which means it significantly perform with IoT infrastructure. Even in zdirection also the coverage is obtained as 200 meters hence the WOA improves the coverage efficiently. Optimal value identified are 15 values where the data can be evaluated for the identification of optimal value for the meta-heuristics approach. For the identified values IoT network provides efficient network performance for improving IoT device communication. Through the proposed approach optimal value identified for the WOA approach is identified as $1.0746 \mathrm{e}-78$ which is considered as an optimal value for the developed IoT infrastructure.

\section{CONCLUSION}

The drastic development of IoT devices provides hyperconnectivity using a cyber-physical-social environment. The devices involved in IoT communication are smart tags. Smartphones, Nano-things, and fewer gadgets of electronics. Also, IoT provides connectivity in a heterogeneous manner to offer effective performance against interface and connectivity for the provision of significant quality of services. In an IoT environment based on user demand services need to be provided for the gathering of appropriate responses for the reception of services which put forth the requirement of effective performance of IoT communication. This user requirement can be achieved through the identification of the optimal path in the network. One other issue related to IoT communication is the management of mobility. Based on this into consideration this research aimed to develop an efficient meta-heuristic approach for improving the overall performance of IoT through a multi-channel routing approach. IoT device communication is subjected to a certain limitation in terms of energy, mobility, and resource management. To improve the performance of IoT communication this research utilizes a meta-heuristics approach known as Whale optimization technique for identification of significant path for transmission of data in the IoT network. The evaluation is performed for consideration of 50 IoT devices for the coverage range of 100 meters. The analysis is performed comparatively with other existing optimization algorithms such as PSO, KHA, firefly, and multi-channel WOA. Simulation results demonstrated that the proposed multi-channel $\mathrm{W}$ effectively exhibits the throughput and packet delivery ratio.

\section{REFERENCES}

[1] Z. Sheng, S. Yang, Y. Yu, A. V. Vasilakos, J. A. McCann, and K. K. Leung. A survey on the IETF protocol suite for the internet of things: Standards, challenges, and opportunities. IEEE wireless communications, vol. 20, no. 6, pp. 91-98, 2013.

[2] F. Rusek et al. Scaling up MIMO: Opportunities and challenges with very large arrays. IEEE signal processing magazine, vol. 30, no. 1, pp. 40-60, 2012

[3] O. Raeesi, J. Pirskanen, A. Hazmi, T. Levanen, and M. Valkama Performance evaluation of IEEE 802.11 ah and its restricted access window mechanism.In 2014 IEEE international conference on communications workshops (ICC), 2014: IEEE, pp. 460-466.

[4] S. Debroy, S. Bhattacharjee, and M. Chatterjee. Spectrum map and its application in resource management in cognitive radio networks. IEEE Transactions on Cognitive Communications and Networking, vol. 1, no. 4, pp. 406-419, 2015

[5] A. A. AlZubi, M. Al-Maitah, and A. Alarifi. A best-fit routing algorithm for non-redundant communication in large-scale IoT based network. Computer Networks, vol. 152, pp. 106-113, 2019.

[6] B. B. Olyaei, J. Pirskanen, O. Raeesi, A. Hazmi, and M. Valkama. Performance comparison between slotted IEEE 802.15. 4 and IEEE 802.1 lah in IoT based applications.In 2013 IEEE 9th International Conference on Wireless and Mobile Computing, Networking and Communications (WiMob), 2013: IEEE, pp. 332-337.

[7] H. Bogucka, P. Kryszkiewicz, and A. Kliks. Dynamic spectrum aggregation for future $5 \mathrm{G}$ communications. IEEE Communications Magazine, vol. 53, no. 5, pp. 35-43, 2015.

[8] K. Mekki, E. Bajic, F. Chaxel, and F. Meyer. A comparative study of LPWAN technologies for large-scale IoT deployment. ICT express, vol. 5, no. 1, pp. 1-7, 2019.

[9] M. A. da Cruz, J. J. Rodrigues, A. K. Sangaiah, J. Al-Muhtadi, and V. Korotaev. Performance evaluation of IoT middle ware. Journal of Network and Computer Applications, vol. 109, pp. 53-65, 2018.

[10] J. Li, B. N. Silva, M. Diyan, Z. Cao, and K. Han. A clustering based routing algorithm in IoT aware Wireless Mesh Networks. Sustainable cities and society, vol. 40, pp. 657-666, 2018.

[11] D. Chemodanov, F. Esposito, A. Sukhov, P. Calyam, H. Trinh, and Z. Oraibi. AGRA: AI-augmented geographic routing approach for IoTbased incident-supporting applications. Future Generation Computer Systems, vol. 92, pp. 1051-1065, 2019.

[12] Z. Han, Y. Li, and J. Li. A novel routing algorithm for IoT cloud based on hash offset tree. Future Generation Computer Systems, vol. 86, pp. 456-463, 2018.

[13] L. Calderoni, A. Magnani, and D. Maio. IoT Manager: An open-source IoT framework for smart cities. Journal of Systems Architecture, vol. 98, pp. 413-423, 2019.

[14] S. Jeon and I. Jung. Experimental evaluation of improved IoT middleware for flexible performance and efficient connectivity. Ad Hoc Networks, vol. 70, pp. 61-72, 2018.

[15] S. Mukherjee and G. Biswas. Networking for IoT and applications using existing communication technology. Egyptian Informatics Journal, vol. 19, no. 2, pp. 107-127, 2018.

[16] V. Casola, A. De Benedictis, M. Rak, and U. Villano. Toward the automation of threat modeling and risk assessment in IoT systems. Internet of Things, vol. 7, p. 100056, 2019.

[17] M. S. Pour, E. Bou-Harb, K. Varma, N. Neshenko, D. A. Pados, and K.-K. R. Choo. Comprehending the IoT cyber threat landscape: A data dimensionality reduction technique to infer and characterize Internetscale IoT probing campaigns/ Digital Investigation, vol. 28, pp. S40S49, 2019.

[18] M. Hasan, M. M. Islam, M. I. I. Zarif, and M. Hashem. Attack and anomaly detection in IoT sensors in IoT sites using machine learning approaches. Internet of Things, vol. 7, p. 100059, 2019.

[19] P. Valerio. Can Sub-1GHz WiFi Solve The IoT Connectivity Issues The New Global Enterprise, 2014.

[20] B. B. Olyaei, J. Pirskanen, O. Raeesi, A. Hazmi, and M. Valkama. Performance comparison between slotted IEEE 802.15. 4 and IEEE 
802.1 lah in IoT based applications. In 2013 IEEE 9th International Conference on Wireless and Mobile Computing, Networking and Communications (WiMob), 2013: IEEE, pp. 332-337.

[21] L. Monostori et al. Cyber-physical systems in manufacturing. Cirp Annals, vol. 65, no. 2, pp. 621-641, 2016.

[22] T. Rault, A. Bouabdallah, and Y. Challal. Energy efficiency in wireless sensor networks: A top-down survey. Computer Networks, vol. 67, pp. 104-122, 2014

[23] K. Mekki, E. Bajic, F. Chaxel, and F. Meyer. A comparative study of LPWAN technologies for large-scale IoT deployment. ICT express, vol. 5, no. 1, pp. 1-7, 2019.

[24] M.-S. Pan and S.-W. Yang. A lightweight and distributed geographic multicast routing protocol for IoT applications. Computer Networks vol. 112, pp. 95-107, 2017.

[25] J. Huang, Q. Duan, Y. Zhao, Z. Zheng, and W. Wang. Multicast routing for multimedia communications in the Internet of Things. IEEE Internet of Things Journal, vol. 4, no. 1, pp. 215-224, 2016.

[26] M. Z. Hasan and F. Al-Turjman. Optimizing multipath routing with guaranteed fault tolerance in Internet of Things. IEEE Sensors Journal, vol. 17, no. 19, pp. 6463-6473, 2017.

[27] D. Rosário, Z. Zhao, A. Santos, T. Braun, and E. Cerqueira. A beaconless opportunistic routing based on a cross-layer approach for efficient video dissemination in mobile multimedia IoT applications. Computer communications, vol. 45, pp. 21-31, 2014.

[28] M. Ishino, Y. Koizumi, and T. Hasegawa. A routing-based mobility management scheme for IoT devices in wireless mobile networks. IEICE Transactions on Communications, vol. 98, no. 12, pp. 23762381, 2015.

[29] A. A. Khan, M. H. Rehmani, and M. Reisslein. Requirements, design challenges, and review of routing and MAC protocols for CR-based smart grid systems. IEEE Communications Magazine, vol. 55, no. 5 , pp. 206-215, 2017.

[30] J. Zhu, Y. Song, D. Jiang, and H. Song. Multi-armed bandit channe access scheme with cognitive radio technology in wireless sensor networks for the internet of things. IEEE access, vol. 4, pp. 4609-4617, 2016.

[31] F. Al-Turjman. Cognitive routing protocol for disaster-inspired internet of things. Future Generation Computer Systems, vol. 92, pp. 1103 1115, 2019.

[32] H. Bogucka, P. Kryszkiewicz, and A. Kliks.Dynamic spectrum aggregation for future $5 \mathrm{G}$ communications. IEEE Communications Magazine, vol. 53, no. 5, pp. 35-43, 2015.

[33] Y. Takizawa, Y. Takashima, and N. Adachi. Self-Organizing Localization for Wireless Sensor Networks Based on Neighbor Topology. In Proc. IARIA UBICOMM 2013, 2013, pp. 102-108.

[34] S. Huaizhou, R. V. Prasad, E. Onur, and I. Niemegeers. Fairness in wireless networks: Issues, measures and challenges. IEEE Communications Surveys \& Tutorials, vol. 16, no. 1, pp. 5-24, 2013.

[35] W. Rehan, S. Fischer, M. Rehan, and M. H. Rehmani. A comprehensive survey on multichannel routing in wireless sensor networks. Journal of Network and Computer Applications, vol. 95, pp. 1-25, 2017.

[36] O. Iova, P. Picco, T. Istomin, and C. Kiraly. Rpl: The routing standard for the internet of things... or is it? IEEE Communications Magazine, vol. 54, no. 12, pp. 16-22, 2016.

[37] B. P. Santos, O. Goussevskaia, L. F. Vieira, M. A. Vieira, and A. A. Loureiro. Mobile Matrix: Routing under mobility in IoT, IoMT, and Social IoT. d Hoc Networks, vol. 78, pp. 84-98, 2018.

[38] B. Afzal, M. Umair, G. A. Shah, and E. Ahmed. Enabling IoT platforms for social IoT applications: vision, feature mapping, and challenges. Future Generation Computer Systems, vol. 92, pp. 718-731, 2019.

[39] L. Atzori, A. Iera, G. Morabito, and M. Nitti. The social internet of things (siot)-when social networks meet the internet of things: Concept, architecture and network characterization. Computer networks, vol. 56, no. 16, pp. 3594-3608, 2012.

[40] O. Gnawali, R. Fonseca, K. Jamieson, M. Kazandjieva, D. Moss, and P. Levis. CTP: An efficient, robust, and reliable collection tree protocol for wireless sensor networks. ACM Transactions on Sensor Networks (TOSN), vol. 10, no. 1, pp. 1-49, 2013.

[41] B. Peres et al. Matrix: Multihop address allocation and dynamic anyto-any routing for 6LoWPAN. Computer Networks, vol. 140, pp. 28 40, 2018.

[42] D. Johnson, C. Perkins, and J. Arkko. Mobility support in IPv6. ed RFC 3775, june, 2004.

[43] M. D. Esfahani, A. A. Rahman, and N. H. Zakaria. Green IT/IS adoption as corporate ecological responsiveness: an academic literature review. Journal of Soft Computing and Decision Support Systems, vol. 2, no. 1, pp. 35-43, 2015.

[44] M.-C. Chuang and J.-F. Lee. FH-PMIPv6: A fast handoff scheme in Proxy Mobile IPv6 networks. In 2011International Conference on
Consumer Electronics, Communications and Networks (CECNet) 2011: IEEE, pp. 1297-1300.

[45] A. J. Jabir, S. Shamala, and Z. Zuriati. A New Strategy for Signaling Overhead Reduction in the Proxy Mobile IPv6 Protocol. American Journal of Applied Sciences 9 (4): 535-541, 2012

[46] S. H. Chae, W. Choi, J. H. Lee, and T. Q. Quek. Enhanced secrecy in stochastic wireless networks: Artificial noise with secrecy protected zone. IEEE Transactions on Information Forensics and Security, vol. 9, no. 10, pp. 1617-1628, 2014

[47] P. N. Mahalle, B. Anggorojati, N. R. Prasad, and R. Prasad. Identity authentication and capability based access control (iacac) for the internet of things. Journal of Cyber Security and Mobility, vol. 1, no. 4, pp. 309-348, 2013

[48] M. Henze, B. Wolters, R. Matzutt, T. Zimmermann, and K. Wehrle. Distributed configuration, authorization and management in the cloudbased internet of things. in 2017 IEEE Trustcom/BigDataSE/ICESS, 2017: IEEE, pp. 185-192.

[49] T. Pecorella, L. Brilli, and L. Mucchi. The role of physical layer security in IoT: A novel perspective. Information, vol. 7 , no. 3, p. 49 , 2016.

[50] H. Yu, M. Kaminsky, P. B. Gibbons, and A. Flaxman. Sybilguard: defending against sybil attacks via social networks. In Proceedings of the 2006 conference on Applications, technologies, architectures, and protocols for computer communications, 2006, pp. 267-278.

[51] J. Mattila. The blockchain phenomenon-the disruptive potential of distributed consensus architectures. ETLA working papers, 2016.

[52] J. Gubbi, R. Buyya, S. Marusic, and M. Palaniswami. Internet of Things (IoT): A vision, architectural elements, and future directions. Future generation computer systems, vol. 29, no. 7, pp. 1645-1660, 2013.

[53] N. Michael and A. Tang. Halo: Hop-by-hop adaptive link-state optimal routing. EEE/ACM Transactions on Networking, vol. 23, no. 6, pp 1862-1875, 2014.

[54] F. J. Rodriguez, S. Fernandez, I. Sanz, M. Moranchel, and E. J. Bueno. Distributed approach for smart grids reconfiguration based on the OSPF routing protocol. IEEE Transactions on Industrial Informatics, vol. 12 , no. 2, pp. 864-871, 2015

[55] S. Debroy, S. De, and M. Chatterjee. Contention based multichannel MAC protocol for distributed cognitive radio networks. IEEE Transactions on Mobile Computing, vol. 13, no. 12, pp. 2749-2762, 2014.

[56] S. Bhattarai, J.-M. J. Park, B. Gao, K. Bian, and W. Lehr. An overview of dynamic spectrum sharing: Ongoing initiatives, challenges, and a roadmap for future research. IEEE Transactions on Cognitive Communications and Networking, vol. 2, no. 2, pp. 110-128, 2016.

[57] I. Yaqoob et al. Internet of things architecture: Recent advances, taxonomy, requirements, and open challenges. IEEE wireless communications, vol. 24, no. 3, pp. 10-16, 2017.

[58] M. Brachmann, O. Garcia-Mochon, S.-L.Keoh, and S. S. Kumar Security considerations around end-to-end security in the IP-based Internet of things. in Workshop on Smart Object Security, in conjunction with IETF83, Paris, France, March 23, 2012, 2012.

[59] J. M. Mohammed, B.-L. Ong, R. B. Ahmad, and M. Hakawati. Internet of things (IoT) mobility support based on distributed sensor proxy mipv6. Journal of Theoretical \& Applied Information Technology, vol 95 , no. 17,2017

[60] P. Kamalinejad, C. Mahapatra, Z. Sheng, S. Mirabbasi, V. C. Leung, and Y. L. Guan. Wireless energy harvesting for the Internet of Things IEEE Communications Magazine, vol. 53, no. 6, pp. 102-108, 2015. 